\title{
Processing of identity and conditional relations in monkeys (Cebus apella) and pigeons (Columba livia)
}

\author{
M. R. D'AMATO, DAVID P. SALMON, ERIC LOUKAS, and ART TOMIE \\ Rutgers-The State University, New Brunswick, New Jersey
}

\begin{abstract}
As a test of differential processing of identity and conditional relations, subjects were first highly trained on 0-sec delay identity matching (IM) and conditional ("symbolic") matching (CM), with the same pair of stimuli serving as samples in both tasks. CM probe trials were then randomly interspersed among baseline IM trials and vice versa. The differences in response latencies obtained on the probe and baseline trials provided the critical data. It was argued that differential processing of identity and conditional relations should result in a larger difference in response latency when CM probes were interspersed among IM baseline trials than when the reverse assignment was made. This pattern was found in cebus monkeys but not in pigeons. The results provide further evidence for the matching concept in monkeys, and they support the view that the matching concept is at best only weakly represented in pigeons.
\end{abstract}

Because of its fundamental nature, the matching concept-also known as the identity or same/different concept-has played an important role in comparative cognition (e.g., Premack, 1978, 1983). Animals appear to differ greatly in the degree to which they are capable of developing the matching concept and in the range of conditions to which the concept is spontaneously applied. As revealed by transfer tests to new stimuli, monkeys can develop the matching concept in the visual modality with training on as few as two exemplars (D'Amato, Salmon, $\&$ Colombo, 1985). But the limited generality of the concept is revealed by the fact that, without additional extensive matching training in the target modality, it does not extend to audition (D'Amato \& Colombo, 1985), or, apparently, to touch (Milner, 1973). And within the visual modality itself, unless extended by further relevant training, the identity concept seems largely limited to the general class of stimuli with which it was developed, at least in cebus monkeys (D'Amato, Salmon, \& Colombo, 1985).

The matching concept seems to be even less well represented in nonmammals. D'Amato, Salmon, and Colombo (1985) argued in some detail that several putative demonstrations of the matching concept in pigeons could be accounted for by simpler (i.e., nonconceptual) processes, and recent failures to find evidence of the matching concept in pigeons lend credence to their anal-

This research was supported by NSF Grants No. 8207146 and 8417383 to' M. R. D'Amato. Preliminary data from Experiment 1 were described by D'Amato and Salmon (1984), who were responsible for the primate work. The pigeon research was the responsibility of E. Loukas and A. Tomie. We are grateful to $M$. Colombo for assistance with the monkey study. Reprints may be obtained from M. R. D'Amato, Department of Psychology, Rutgers-The State University, New Brunswick, NJ 08903. ysis (Wilson, Mackintosh, \& Boakes, 1985a, 1985b). A report of the matching concept in goldfish (Zerbolio \& Royalty, 1983) was similarly interpreted by D'Amato, Salmon, and Colombo (1985), and a subsequent followup study supported their view (Zerbolio, 1985).

In most previous relevant studies, representation of the matching concept has been inferred from the performance obtained on transfer tests with novel sample stimuli. This strategy presents little difficulty if the test stimuli are truly new (ideally, orthogonal to the training stimuli) and the transfer performance is very high. Thus, if an animal trained to match red and green disks continued to perform at a high level when tested with two forms, say, a circle and a horizontal line, it would be reasonable to infer some degree of representation of the matching concept. But if the test stimuli were orange and blue-green disks, a high performance level on the transfer test might be due to stimulus generalization rather than to conceptmediated transfer. As the number of training stimuli that precede the transfer test increases, this interpretative problem takes on added significance because of the expanded opportunity for stimulus generalization from previous sample sets.

On the other hand, introduction of novel stimuli often disrupts an animal's performance, which may complicate interpretation of the transfer results gotten during the critical first session or so. In some species, the novel stimuli may generate long-lasting position or stimulus preferences, and although this result could be taken as evidence that the matching concept is not well represented in the species, it prevents unambiguous assessment of the latter possibility.

D'Amato and Salmon (1984) pointed out that there are other operations from which representation of the matching concept can be inferred. A species in which the match- 
ing concept is absent or is weakly represented very likely learns identity matching (IM) and conditional, or symbolic, matching (CM) tasks in much the same way (Carter \& Werner, 1978). In such a species, one would expect transfer between IM and CM tasks to be bidirectional, which is to say, previous training on a series of either IM or CM tasks ought to facilitate subsequent learning of both IM and CM. On the other hand, a species in which the identity concept is well represented will ordinarily process identity and conditional relations in different ways, inasmuch as there is no general rule that governs performance in CM tasks. Transfer between IM and CM tasks will not be bidirectional in such a species, and it is even possible that negative transfer will be observed when the animal is shifted from IM to $\mathrm{CM}$.

Working with cebus monkeys, D'Amato, Salmon, and Colombo (1985) reported that previous CM training did not result in the massive amounts of positive transfer to an IM task that were produced by a comparable amount of previous IM training. Moreover, they observed that monkeys sophisticated with regard to IM tend to have considerable difficulty learning a CM task. Although the relevant data are sparse, transfer between IM and CM seems to be largely bidirectional in pigeons. After being trained on a number of IM problems, Holmes's (1979) pigeons showed a high degree of transfer to both IM and CM tasks (see Wilson et al., 1985a, 1985b).

In the present studies, we examined another possible difference in the way identity and conditional relations are processed by monkeys and pigeons. Subjects were trained on an IM and a CM task in which the samples (standard stimuli) were the same pair of stimuli, for example, a red disk and a vertical line. In the case of IM, the comparison stimuli were, of course, also a red disk and a vertical line; for the CM task, they were an inverted triangle and a dot. The animals were trained with 0 -sec delay until IM and CM response latencies were comparable. They were then given a number of IM sessions, followed by critical test sessions in which occasional CM probe trials were interspersed among the IM baseline trials. Note that in these test sessions, the nature of a specific trial, whether IM or CM, was unknown to the subjects until the comparison stimuli appeared. The same procedure was followed for IM probe trials superimposed on a CM baseline.

Our hypothesis was that if the subjects process identity and conditional relations in much the same way, their response latencies on probe and baseline trials should show similar patterns on both types of test sessions. Thus, if latencies on $\mathrm{CM}$ probe trials were elevated over the latencies on the IM trials with which they were interspersed, the latencies of IM probes should be similarly increased over the latencies of baseline CM trials. On the other hand, if identity and conditional relations are processed differently, the former on a conceptual basis and the latter by associations between samples and comparison stimuli, the pattern of latencies obtained during the test sessions would depend on the nature of the probes.
Specifically, the response latencies of CM probe trials would be elevated with regard to the IM baseline latencies, whereas the latencies of IM probes would not be greater than CM baseline latencies, or would be elevated to a much lesser extent.

The basis for this expectation is as follows. A series of CM sessions should encourage the animal to anticipate the correct comparison stimulus while the sample is still present, a form of "prospective" processing (Honig \& Thompson, 1982). Because the animal has already retrieved the correct stimulus before the comparison stimuli appear, prospective processing will result in shorter response latencies than would be possible without such prior processing. When, on the other hand, $\mathrm{CM}$ trials occur as probes against an IM baseline, the correct $\mathrm{CM}$ comparison stimulus is not likely to be anticipated during the sample presentation period; indeed, if the animal did engage in prospective processing, it would result in anticipation of the correct IM comparison stimulus. In either case, response latencies on $\mathrm{CM}$ probes will be elevated compared to latencies on baseline CM or baseline IM trials.

Identity matching that is governed by a single rule (the matching concept) has little to gain from prospective processing, inasmuch as it is not based on separate associations, as is the case for CM. The sample itself defines the correct comparison stimulus. Still, one might expect response latency on IM probes to suffer somewhat because the animal, anticipating the correct $\mathrm{CM}$ comparison stimulus, would suddenly be confronted with the IM comparison stimuli and would have to shift to an IM mode. Nevertheless, the animal is in a better position on IM probe trials than on CM probe trials, because although both involve a shift in the mode of matching, on CM probe trials the animal must retrieve the correct comparison stimulus as well. Consequently, the difference between response latencies on CM probe and IM baseline trials ought to be greater than the difference between response latencies on IM probe and CM baseline trials.

\section{EXPERIMENT 1}

The first experiment employed monkeys as subjects. Because of the extensive training and testing required and the need to run a control condition after Phase 1 was completed, almost 4 years elapsed between the beginning and the completion of the study.

\section{Method}

\section{Subjects}

Two male (Moe and Roscoe) and 3 female (Coco, Dagwood, and Fifi) Cebus apella monkeys served as subjects. Moe, the only laboratory-born subject, was 3 years of age at the start of the study; the other monkeys were between 16 and 19 years of age. Moe's previous training with visual stimuli was limited to two conditional matching problems followed by two identity matching tasks (D'Amato, Salmon, \& Colombo, 1985; D'Amato, Salmon, Loukas, \& Tomie, 1985). The other subjects had had extensive experience 
with visual identity matching. Coco and Fifi had substantial previous experience with conditional (symbolic) matching (D'Amato \& Worsham, 1974), Roscoe had just completed a study involving conditional matching (D’Amato, Salmon, Loukas, \& Tomie, 1985), and Dagwood had been trained on a great variety of experimental tasks, including conditional discriminations.

The monkeys were housed in individual cages, with water constantly available. Food (Purina Monkey Chow No. 5045) was restricted to a single feeding ( 1 to $2 \mathrm{~h}$ following an experimental session), adjusted to the maximum amount that supported reliable performance in each monkey. The daily rations were sufficient to maintain the animals at $90 \%-100 \%$ of their free-feeding body weights

\section{Apparatus}

Two monkey test chambers were used. The front wall of each chamber accommodated four inline projectors located at the four corners of a $12-\mathrm{cm}$ square, with a fifth projector at the center of the square. Each projector was fitted with a transparent key that served as the response mechanism. Only the center and top two projectors were used; the sample stimuli appeared on the former and the comparison stimuli on the latter. A microswitch, used to initiate a trial, was located below the projector array; below the microswitch was a recessed dipper well that could be illuminated to signal the intertrial interval (ITI). Noyes 190 -mg banana pellets served as reinforcers and were delivered to a food cup located on the right wall.

Illumination of the chamber was provided by an overhead houselight, a 25-W soft-white bulb located behind a translucent shield. The houselight could be dimmed by adding a $500-\Omega$ resistor in series with the bulb. Stimulus presentation, programming of trial events, and data recording were accomplished by a PDP 8/e computer and a Commodore disk unit. Response latencies were measured to the nearest 20 msec.

The stimuli consisted of a red disk that illuminated a circular area approximately $25 \mathrm{~mm}$ in diameter on the projector, and three forms-an inverted triangle, a vertical line, and a dot. All forms appeared as white figures on a black background and, except for the dot, were composed of white lines, approximately $1.5 \mathrm{~mm} \times$ $17 \mathrm{~mm}$. The dot was $6 \mathrm{~mm}$ in diameter.

\section{Procedure}

Phase 1: Training. Because of their previous experience with identity matching, the subjects required very little training on the IM task. The CM stimulus assignments of Phase 1 are shown in Table 1, and, of course, for each monkey the samples and the comparison stimuli of its IM task were the same stimuli that served as samples in the CM task.

Table 1 shows that for Coco, Dagwood, and Moe the assigned samples were the same, as were the comparison stimuli; however,

Table 1

Stimulus Assignments for the Conditional Matching Task of Phase 1, Experiment 1

\begin{tabular}{rrr}
\hline Subject & \multicolumn{1}{c}{ Samples } & Comparison Stimuli \\
\hline Coco & Inverted Triangle & $\rightarrow$ Red \\
Dot & $\rightarrow$ Vertical Line \\
Dagwood & Inverted Triangle & $\rightarrow$ Red \\
Dot & $\rightarrow$ Vertical Line \\
Moe & Inverted Triangle & $\rightarrow$ Vertical Line \\
Dot & $\rightarrow$ Red \\
Red & - Inverted Triangle \\
Fifi & Vertical Line & - Dot \\
Roscoe & Red & - Dot \\
& Vertical Line & - Inverted Triangle
\end{tabular}

the conditional pairing of samples and comparison stimuli used for Moe differed from that used for Coco and Dagwood. The samples and comparison stimuli assumed reverse roles in the case of Fifi and Roscoe, and the conditional pairing of samples and comparison stimuli differed for these 2 subjects.

Moe and Roscoe had recently received substantial training on their CM tasks (D'Amato, Salmon, Loukas, \& Tomie, 1985), so that acquisition training was unnecessary. Coco, Fifi, and Dagwood required 432,264 , and 552 trials, respectively, to satisfy the criterion of two consecutive 24-trial sessions with at least $90 \%$ correct responses.

The goal of the next stage of training was to provide each subject with the additional IM and CM experience needed to produce consistently high levels of accuracy on both tasks and reasonably comparable response latencies. This required from 25 (Roscoe) to 76 (Moe) 24-trial sessions of CM training and from 2 (Dagwood) to 58 (Moe) sessions of IM training.

During this additional training, the stimulus parameters and trial procedures to be employed during testing were introduced. These were as follows. A trial was initiated by pressing the microswitch 10 times, which resulted in a .4-sec tone that signaled presentation of the sample stimulus on the center key. The sample duration was fixed ( $1 \mathrm{sec}$, in most cases), and after its termination the comparison stimuli appeared on the upper two projectors $(0$-sec delay matching). A single press on the response key that displayed the correct comparison stimulus resulted in termination of the stimulus display, delivery of a food pellet, and entry into a 20 -sec ITI, during which the houselight was dimmed and the dipper well was illuminated. A press on the response key that presented the incorrect comparison stimulus terminated the stimulus display and began a $60-\mathrm{sec}$ timeout period, signaled by the dim houselight alone; the ITI followed the timeout.

On each trial, response latency was measured from the onset of the comparison stimuli until a response was executed. Virtually all sessions consisted of 24 trials, each sample stimulus appearing 12 times in a quasi-random order. Normally, a single session was given on each of the 6 days per week that the experiment was conducted.

Phase 1: Testing. After the completion of training, a series of test cycles was begun. A test cycle consisted of a series of 24-trial pretest sessions, all with the same type of trials (IM or CM), the point of which was to establish an IM or CM "set." This was followed by 1028 -trial test sessions in which a small number of probes were intermixed with the baseline trials. There were four probe trials in each test session, distributed randomly over the last 24 of the 28 trials, with the restriction that only one probe could occur in each block of 6 trials. The probes were CM trials when the baseline trials were IM, and vice versa. Thus, in an IM/CM test cycle, the subject received a series of pretest IM sessions followed by 10 sessions in which 4 of the 28 trials were probe $\mathrm{CM}$ trials; a CM/IM cycle consisted of a series of $\mathrm{CM}$ sessions followed by 10 test sessions composed of $4 \mathrm{IM}$ probe trials and $24 \mathrm{CM}$ baseline trials.

Test sessions did not begin until the subject's response latencies were reasonably stable in the pretest sessions. The number of pretest sessions received prior to the 10 test sessions of a test cycle averaged 11.3 for the IM/CM cycles and 11.1 for CM/IM; no subject received fewer than 8 such sessions in any cycle.

Four of the subjects completed four test cycles, two each of IM/CM and CM/IM, which alternated; Roscoe received only one test cycle of each kind. Three subjects began with $\mathrm{IM} / \mathrm{CM}$ and 2 with CM/IM. Sample duration was 1 sec during all pretest and test sessions, except for Coco and Fifi, the lead animals in the study. Sample duration was $1.5 \mathrm{sec}$ for Fifi and for Coco's first two test cyles; it was reduced to $.3 \mathrm{sec}$ for Coco's last two cycles.

Phase 2: Training. Coco, Fifi, and Dagwood participated in this phase; Moe and Roscoe were committed to other experiments. The subjects were retrained on the CM task, with the roles of samples and comparison stimuli reversed from what they had been in Phase 1 (Table 1). The same stimuli were conditionally related, however. 
Thus, Coco was trained in Phase 2 with the inverted triangle as the correct comparison stimulus for the red disk sample, and the dot as the correct comparison stimulus for the vertical line sample.

Between completion of Phase 1 and the beginning of Phase 2, all 3 monkeys participated in a study of backward associations that involved the CM tasks of Phase 1 (D'Amato, Salmon, Loukas, \& Tomie, 1985). Despite the enormous amount of experience that the subjects had amassed with the CM discriminanda, and notwithstanding the fact that the stimuli that were conditionally paired in Phase 1 were also paired in Phase 2 (albeit in the reverse direction), learning was slow. Coco, Dagwood, and Fifi required 55, 45, and 34 24-trial sessions, respectively, to reach the same acquisition criterion as was employed in Phase 1 . This slow acquisition is further indication of the insignificance of backward associations in the process ing of conditional relations by monkeys and other animals (D'Amato, Salmon, Loukas, \& Tomie, 1985).

An additional 14, 13, and 47 24-trial sessions of $\mathrm{CM}$ were given to Coco, Dagwood, and Fifi, respectively, to make their $\mathrm{CM}$ response accuracy and latencies comparable to their IM performances.

Phase 2: Testing. Apart from the changes in the $\mathrm{CM}$ associative relationships, the Phase 2 test cycles were conducted in the same manner as those of Phase 1-a series of pretest sessions followed by 10 test sessions. The mean number of pretest sessions received prior to the 10 test sessions of a test cycle was 10.3 for the IM/CM cycles and 11.6 for the CM/IM cycles. Each of the 3 subjects that were run in this phase received four test cycles, with $\mathrm{IM} / \mathrm{CM}$ and $\mathrm{CM} / \mathrm{IM}$ cycles alternating. The initial test cycle was CM/IM for 2 subjects; IM/CM was first for the third. Sample duration was $1 \mathrm{sec}$ throughout Phase 2.

\section{Results}

\section{Phase 1}

Response latencies on the IM and CM pretest sessions that preceded the test sessions were quite comparable. For each subject, a median latency was obtained for each of the last eight IM pretest sessions of the IM/CM cycles, which were then averaged to obtain a single IM latency value for each subject; the same was done for the last eight CM pretest sessions. The group mean latencies based on these summary scores turned out to be identical for IM and CM $(.73 \mathrm{sec})$. The mean percentage of correct responses during the eight pretest sessions was also very similar for IM and CM (96.2 vs. 96.6).

Each of the 10 test sessions of a cycle generated two median latencies for each subject: one based on the last 20 baseline trials and the other on the 4 interspersed probe trials. Subjects that received two IM/CM and CM/IM test cycles had their two sets of latencies averaged, resulting in a single baseline and a single probe latency for each subject on each of the 10 sessions of the IM/CM and $\mathrm{CM} / \mathrm{IM}$ cycles. Figure 1 presents group means based on these latencies.

Before we discuss the latency data, it should be noted that during the test sessions response accuracy on IM and CM trials was high and comparable. The mean percentage of correct responses on the IM baseline trials of the IM/CM cycles was 97.0 ; it was 97.4 for the CM baseline trials of the $\mathrm{CM} / \mathrm{IM}$ cycles. The corresponding values for the CM and IM probe trials were $92.0 \%$ and $93.4 \%$, respectively.

Figure 1 clearly shows that, in spite of comparable latencies on the IM and CM baseline trials of the test sessions (left and right panels, respectively), the latencies of CM probes (left panel) were much more elevated than those of IM probes (right panel). Moreover, referring to the left panel, the difference between the latencies on CM probe and IM baseline trials was somewhat larger when averaged over the last two testing sessions of the IM/CM cycle than when averaged over the first two; in contrast, the largest difference between probes and baseline trials

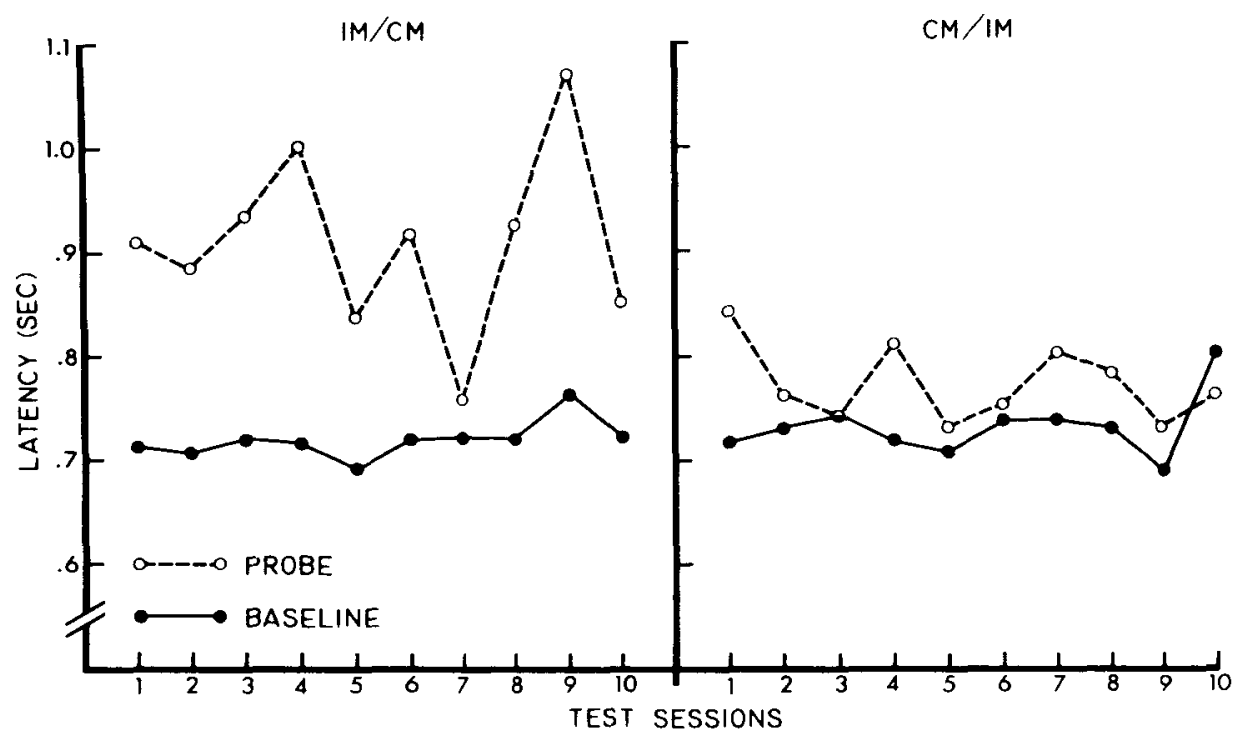

Figure 1. Group mean response latencies on baseline and probe trials during the test sessions of Phase 1 of Experiment 1 . In the IM/CM cycle (left panel), the baseline trials were identity matching (IM) trials and the probes were conditional matching (CM) trials; in the CM/IM cycle (right panel), baseline trials were CM and probe trials were IM. The subjects were 5 cebus monkeys. 
in the CM/IM test cycles (right panel) occurred in the first test session, essentially vanishing by the end of testing.

Separate randomized-blocks ANOVAs (Kirk, 1968), based on subjects as blocks (5), trial type [probe vs. baseline trials (2)], and test sessions (10), were applied to the data generated by the IM/CM and CM/IM cycles. The difference between probe and baseline trials was highly reliable for the IM/CM cycle $[F(1,76)=47.76$, $p<.001]$. The corresponding comparison for the $\mathrm{CM}$ /IM cycle was also statistically significant, but at a much lower level of reliability $[F(1,76)=5.17$, $p<.05$ ]. Neither test sessions nor the trial type $\times$ test sessions interaction was statistically significant in either cycle. An overall randomized-blocks ANOVA, which included cycles as a factor, showed the trial type $\times$ cycles interaction to be significant $[F(1,156)=18.96$, $p<.001]$. This result indicates that the difference between probe and baseline latency in the IM/CM cycle was reliably larger than the corresponding difference in the $\mathrm{CM} / \mathrm{IM}$ cycle.

Statistical analyses were also performed on the individual subjects' data. As described above, a test session generated, for each subject, one median latency for the 20 baseline trials and another for the 4 probe trials. A difference score was formed from each such pair of median latencies, and a Wilcoxon matched-pairs signedranks test (Siegel, 1956) was applied to the 10 difference scores that were produced by each test cycle. There were a total of 18 such tests, 9 each for the IM/CM and CM/IM test cycles. (Roscoe contributed two tests, the other subjects, four each.)

At a two-tail .05 level of significance, in six of the nine IM/CM cycles, CM probe latency was significantly longer than baseline IM latency; the difference was not significant in the other three. For the nine CM/IM cycles, IM probe latencies were significantly longer than CM baseline latencies in three cases, nonsignificantly different in five cases, and significantly shorter in one. The three failures to find significantly higher $\mathrm{CM}$ probe latencies in the IM/CM cycles were confined to Dagwood (two) and Moe; these subjects also accounted for two of the three instances in the CM/IM cycles in which probe IM latencies were significantly longer than baseline $\mathrm{CM}$ latencies.

To summarize these individual-subject analyses, during the test sessions Fifi, Roscoe, and Coco produced the pattern of probe and baseline latencies expected on the assumption that they were processing IM and CM in fundamentally different ways; the evidence for Moe was somewhat less convincing. Dagwood provided virtually no evidence of differential processing.

Examination of the individual subjects' data suggested that the red disk and vertical line may have been a slightly more discriminable pair of stimuli than were the inverted triangle and dot, in that response latencies tended to be somewhat shorter on the matching task that employed the former pair as comparison stimuli. Such an asymmetry in discriminability may have reduced the evidence for differential processing of IM and CM relations in susceptible subjects that were assigned the inverted triangle and dot as the sample stimuli (Coco, Dagwood, and Moe, Table 1). It was for this reason that the samples and comparison stimuli of Phase 1 had their roles interchanged in Phase 2.

Phase 2. Coco, Dagwood, and Fifi participated in this phase. Response latencies on the last eight pretest sessions were again closely comparable for IM and CM (.71 vs. $.73 \mathrm{sec})$, as was response accuracy $(98.7 \%$ vs. $98.0 \%)$. Response accuracy remained high and reasonably comparable during the test sessions, averaging $99.7 \%$ and $96.0 \%$ during the IM and CM baseline trials, respectively, and $92.3 \%$ and $99.7 \%$ during the CM and IM probe trials.

Figure 2 presents the group probe and baseline latencies during the 10 test sessions of the IM/CM and CM/IM cycles. Probe and baseline latencies on each test session were computed for each subject in the same manner as in Phase 1. As in the previous phase, response latencies were similar on the IM and CM baseline trials of the test sessions. The latencies of CM probes were again consistently higher than IM baseline latencies (left panel), and the size of the difference changed little over the 10 test sessions. In marked contrast, the latencies of IM probes and CM baseline trials (right panel) were quite similar in most test sessions, rendering statistical analysis of the CM/IM test cycle unnecessary. A randomized-blocks ANOVA applied to the latencies of the IM/CM test cycle showed the latency difference between probe and baseline trials to be highly reliable $[F(1,38)=45.33$, $p<.0011$. Neither sessions nor the trial type $\times$ sessions interaction was significant.

Analyses of the individual test cycles based on the Wilcoxon test used in Phase 1 revealed that in all six cases the latencies of CM probe trials were significantly longer than those of the IM baseline trials with which they were interspersed in the IM/CM test cycles. In contrast, in four of the six CM/IM cycles the latencies of IM probes were not significantly different from the latencies of CM baseline trials, and in the other two cases the latencies of IM probes were significantly shorter than $\mathrm{CM}$ baseline latencies. Thus, the latency patterns produced by all 3 subjects in the test cycles are consistent with differential processing of $\mathrm{IM}$ and $\mathrm{CM}$.

\section{Discussion}

The results of Phases 1 and 2 provide strong evidence that our monkeys process identity and conditional relations in different ways. Conditional matching involves associations between samples and comparison stimuli that are unnecessary in identity matching. Thus, to perform successfully on a CM task, upon viewing the sample the monkey must retrieve the correct comparison stimulus and then distinguish it from the other comparison stimulus (or stimuli). In identity matching, only the latter process is involved, as the conceptually based matching rule defines the correct comparison stimulus for all samples. If we assume that on CM probe trials the monkeys did not begin to retrieve the correct comparison stimulus until the com- 


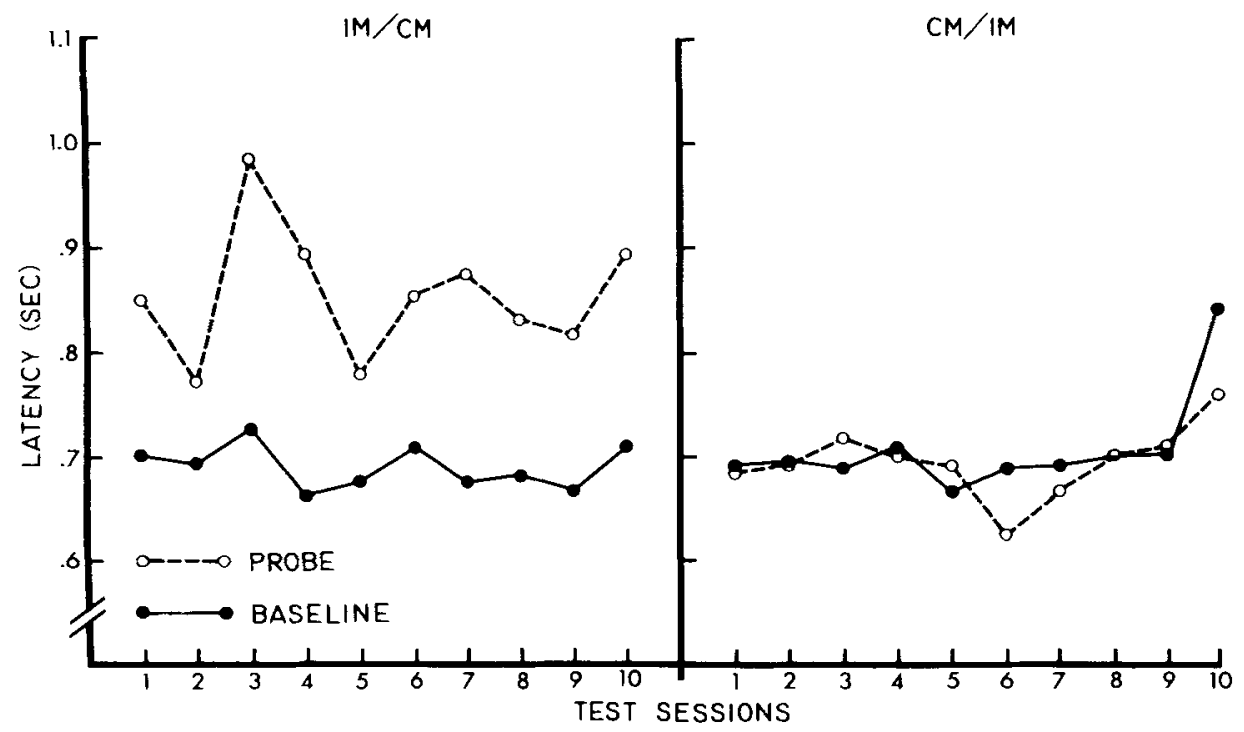

Figure 2. Group mean response latencies on baseline and probe trials during the test sessions of Phase 2 of Experiment 1. In the IM/CM cycle (left panel), the baseline trials were identity matching (IM) trials and the probes were conditional matching (CM) trials; in the CM/IM cycle (right panel), baseline trials were $\mathrm{CM}$ and probe trials were IM. The subjects were 3 cebus monkeys.

parison stimuli appeared, .18 sec, which is the difference between CM probe and IM baseline latencies averaged over Phases 1 and 2, is a reasonable upper-bound estimate of this retrieval time.

There was very little difference between IM and CM response latencies on the baseline trials of the pretest and the test sessions, which may seem inconsistent with the previous discussion. The obvious explanation of these comparable latencies is that the monkeys engaged in prospective processing on the CM baseline trials, retrieving the correct comparison stimulus while the sample was still present (Honig \& Thompson, 1982). Indeed, the point of the pretest sessions was to create the expectation of CM (or IM) trials, and thus encourage such prospective processing. Even with the shortest sample duration employed $(.3 \mathrm{sec})$, there was sufficient time during the CM baseline trials for retrieval of the correct target stimulus before the onset of the comparison stimuli.

It is perhaps a bit surprising that, without the benefit of any kind of physical restraint, small but highly reliable and reproducible differences in response latencies were obtained during the test sessions. We attribute this success largely to the fact that the monkeys were heavily overtrained on the matching tasks, and to the use of only two projectors on which the comparison stimuli could appear. Clearly, as has long been the case for human cognition, under appropriate conditions response latency can be a sensitive indicator of cognitive processes in unrestrained animals.

\section{EXPERIMENT 2}

A parallel study was undertaken with pigeons as subjects, employing reasonably comparable experimental procedures.

\section{Method}

\section{Subjects}

Four experimentally naive male White Carneaux pigeons, approximately 2 years old at the start of the study, served as subjects. They were housed in individual cages, with water and grit continuously available. Throughout the experiment the birds were maintained at $75 \%$ of their free-feeding body weights by supplemental feedings of mixed grain.

\section{Apparatus}

Two pigeon chambers, measuring $35 \times 35 \times 30 \mathrm{~cm}$, were used. Three inline projectors (IEE No. 7835) were mounted on the front wall, $20 \mathrm{~cm}$ above the floor of the chamber; the side projectors were separated from the center unit by $35 \mathrm{~cm}$. Each projector was fitted with a transparent pecking key, which served as the response mechanism. A food hopper was located below the center key, $3 \mathrm{~cm}$ above the floor of the chamber. Ambient illumination was provided by a houselight (two Sylvania $120-\mathrm{W}$ bulbs) mounted behind a strip of translucent plastic that ran the length of the intelligence panel at its top edge. An exhaust fan provided ventilation and was a source of masking noise. Stimulus presentation, programming of trial events, and data recording were controlled by Commodore microcomputers (Model 2001).

Except for small differences in size, the stimuli were the same as those employed in Experiment 1 -a red disk that illuminated a circular area $29 \mathrm{~mm}$ in diameter on the response keys, and three forms: an inverted triangle, a vertical line, and a dot. As projected, the forms appeared as white lines, approximately $2 \mathrm{~mm} \times 17 \mathrm{~mm}$, on a black background. The diameter of the dot was $7 \mathrm{~mm}$.

\section{Procedure}

Training. In the course of 1260 -trial sessions, the pigeons were first autoshaped to peck the three keys, employing the stimuli that were to be used in the next stage of training, which was identity matching. The red disk and vertical line served as the IM stimuli for 2 subjects; for the other 2 , the inverted triangle and dot constituted the sample set.

The beginning of a matching trial was signaled by onset of the houselight and illumination of the center key with white light. A 
single peck on the center key caused the white light to be replaced by one of the sample stimuli. After the completion of 10 pecks (fixedratio [FR] 10) on the sample stimulus, the comparison stimuli appeared on the side keys. In simultaneous matching, the sample remained on the center key; in $\mathbf{0 - s e c}$ delay matching, it was removed. A single peck on the correct comparison stimulus caused the houselight and discriminative stimuli to be extinguished and allowed access to mixed grain in the food hopper for a 4-sec period. A peck on the incorrect comparison stimulus had the same consequences except that food was withheld. A 30-sec ITI followed each trial. Response latency (in units of .1 sec) was measured from the onset of the comparison stimuli until a response was executed. Time to complete the FR 10 on the center key was also recorded.

Training sessions generally consisted of $\mathbf{4 8}$ trials. Each sample stimulus appeared an equal number of times in each session and each comparison stimulus was presented an equal number of times on the left and right keys.

During simultaneous IM training, the criterion of one session with at least $90 \%$ correct responses (equivalent to the two-24trial-sessions criterion used with the monkeys) was satisfied within 4 to 10 sessions. Additional simultaneous training was given, bringing the total of such training to 48-56 sessions for the four birds. This was followed by 18 to 26 sessions of 0 -sec delay IM matching.

The subjects were then placed on the CM task. Each of the 4 birds received one of the four different assignments of comparison stimuli and conditional relations shown in Table 1. Thus the pairs of stimuli that served as samples for the IM task, as well as the conditional relations of the CM task, were balanced over the 4 subjects. The subjects received from 16 to 23 sessions of simultaneous $\mathrm{CM}$, followed by 17 to 230 -sec delay sessions. The criterion of one session with at least $90 \%$ correct was reached in the simultaneous CM task within 3 to 17 sessions.

There then followed 78-85 sessions in which the IM and CM tasks alternated, initially on the basis of one IM session to three CM sessions. During the last 60-62 sessions, which were designed to accustom the pigeons to frequent shifts in the matching task, the alternation was one to one. At the end of this training the subjects had accumulated a total of 124 IM sessions and from 115 to 117 sessions of $\mathrm{CM}$.

In the final two sessions of training, the subjects were familiarized with the impending test sessions. There were 52 trials in a session, consisting of 44 baseline trials and 8 probe trials. Starting with the fifth trial, one probe trial occurred in each block of six trials. The first of these sessions contained $\mathrm{IM}$ baseline trials and CM probes (as in IM/CM test sessions) and the second had the reverse assignment (CM/IM). The latencies on $\mathrm{IM}$ and $\mathrm{CM}$ baseline trials were comparable, differing by $.2 \mathrm{sec}$ for 1 subject and by $.1 \mathrm{sec}$ or less for the other 3 subjects.

Testing. Each subject received one IM/CM and one CM/IM test cycle. The IM/CM cycle came first for 2 subjects and was second for the other 2 . In all cycles, eight (48-trial) pretest sessions preceded the 10 (52-trial) test sessions. The latter had the same composition of baseline and probe trials as did the final two training sessions. Throughout training and testing, which lasted about 12 months, the birds were normally run 6 days per week, one session per day.

\section{Results and Discussion}

Response latency and accuracy were comparable on the eight pretest sessions that preceded a test cycle. The average latency during these sessions was $.74 \mathrm{sec}$ for IM pretest sessions and $.71 \mathrm{sec}$ for CM pretest sessions; the corresponding mean percentages of correct responses were 93.3 and 97.3 . Response accuracy remained comparable during the test sessions, averaging $93.0 \%$ and 97.0\% for the baseline IM and CM trials, respectively, and $96.5 \%$ and $92.3 \%$ for the associated $\mathrm{CM}$ and IM probe trials.

Mean response latencies on the baseline and probe trials of the test sessions are shown in Figure 3. There are a number of differences between these results and those of

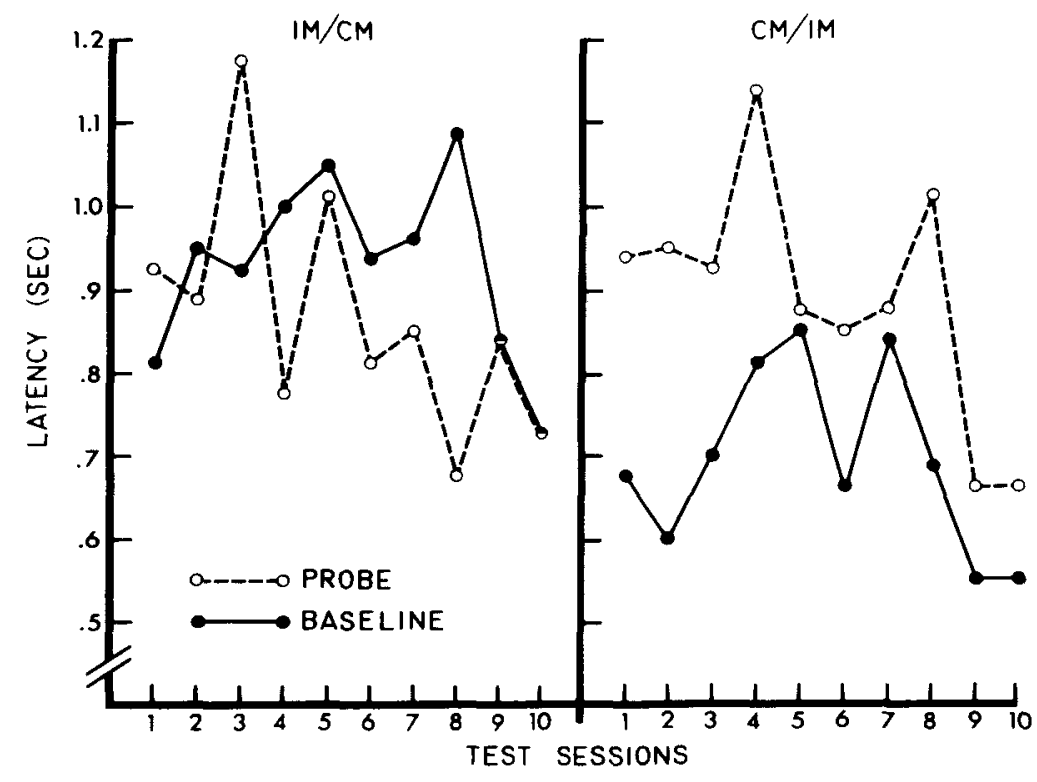

Figure 3. Group mean response latencies on baseline and probe trials during the test sessions of Experiment 2. In the $\mathrm{IM} / \mathrm{CM}$ cycle (left panel), the baseline trials were identity matching (IM) trials and the probes were conditional matching (CM) trials; in the $\mathrm{CM} / \mathrm{IM}$ cycle (right panel), baseline trials were $\mathrm{CM}$ and probe trials were $\mathrm{IM}$. The subjects were 4 pigeons. 
Experiment 1 . In both test cycles, the variability of baseline response latencies across the 10 test sessions was much greater than was the case for the monkey subjects (Figures 1 and 2). Another difference is that the comparable IM and CM latencies observed in the pretest sessions did not, as in the case of the monkeys, continue through the baseline trials of the test sessions; the mean latencies on these IM and CM baseline trials were .93 and $.70 \mathrm{sec}$, respectively. Finally, unlike the pattern of probe-baseline latencies obtained with the monkeys, there was little difference between the latencies obtained on CM probe and IM baseline trials in the IM/CM test cycle, and the latencies of IM probes were elevated over CM baseline latencies in the CM/IM cycle.

The randomized-blocks ANOVA that was employed in Experiment 1 showed that trial type, sessions, and the trial type $\times$ sessions interaction were not significant in the IM/CM test cycle. In the CM/IM cycle, only trial type was significant $[F(1,57)=8.23, p<.01]$.

The individual subjects' data were analyzed by Wilcoxon matched-pairs signed-ranks tests. For the IM/CM cycle, in two cases the latencies of probe CM trials were significantly shorter than those of the baseline IM trials. In the other two cases, probe trial latencies were somewhat longer than baseline latencies, but the differences were far from significant. For the CM/IM cycle, the same 2 subjects that had significantly shorter probe latencies in the IM/CM cycle had significantly longer latencies on the probe IM trials than on the baseline CM trials. For the other 2 subjects, the differences were nonsignificant, with probe latencies again somewhat longer than baseline latencies.

Sample duration was fixed in Experiment 1, whereas in the present study, in order to encourage attending to the sample stimulus, it was subject-controlled. As it turned out, sample exposure duration was generally comparable on IM and CM matching trials. For example, during the eight pretest sessions of the IM/CM and the CM/IM cycles, the mean sample duration was 3.24 and $3.37 \mathrm{sec}$ for the IM and CM trials, respectively.

The results of the present experiment provide no evidence for differential processing of identity and conditional relations in pigeons, at least not of the sort that has been proposed for monkeys. Indeed, the only statistically significant differences in latencies on IM baseline and CM probe trials were opposite in direction from those obtained with the monkeys.

These differences are likely due to specific stimulus and response factors. For example, nonreinforced observing responses to a sample stimulus tend to cause pigeons to avoid comparison stimuli that are similar to the sample, which often results in faster learning of oddity matching than of identity matching. The pattern of probe and baseline latencies produced by the pigeons (Figure 3 ) is probably due, at least in part, to this response tendency. It is interesting in this connection that, unlike the monkeys, the pigeons consistently performed somewhat less accurately on IM than on CM. The same response tendency has been implicated in several putative demonstrations of the matching concept in pigeons (D'Amato, Salmon, \& Colombo, 1985), and it is becoming increasingly clear that the contribution of specific stimulus and response dispositions must be closely considered when analyzing cognitive-like behaviors in animals (cf. Herrnstein, 1985).

Another possible interpretation of the significant difference between the latencies of IM probes and CM baseline trials in the CM/IM cycle is that after prospective processing of the sample stimulus was completed, the pigeons no longer retained the identity of the sample. Thus, when confronted with the comparison stimuli on an IM probe trial, they had to retrieve the appropriate sample stimulus by means of a backward association based on the available CM stimulus. Two facts argue strongly against such an interpretation. First, one would expect from this view a lower level of accuracy on IM probe trials than on IM baseline trials, because the latter do not involve backward association. In fact, as reported above, accuracy on IM probes $(92.3 \%$ correct) was virtually the same as on IM baseline trials $(\mathbf{9 3 . 0 \% )}$. Second, as mentioned earlier, the currently available evidence indicates that backward associations are very weak and evanescent in pigeons and monkeys, if they occur at all (see D'Amato, Salmon, Loukas, \& Tomie, 1985).

\section{GENERAL DISCUSSION}

The results obtained in Experiment 1 clearly indicate that our monkeys process identity and conditional relations in different ways. An associative component is involved in their processing of each conditional relation; based on a single rule, a corresponding process is absent in identity matching. Although monkeys process identity and conditional relations differently, Experiment 2 provided no evidence that this is true for pigeons. Apparently, pigeons process identity relations in much the same way as they process conditional relations, forming an association between each sample and its correct comparison stimuli even in the case of IM (Carter \& Werner, 1978). An appreciation for the abstract quality of "sameness" seems to be either completely lacking or very poorly developed in pigeons (Wilson et al., 1985a, 1985b), although other avian species (corvids) may prove more talented in this regard (Wilson, et al., 1985b). It is thus understandable why it has proven difficult to obtain unambiguous evidence for concept-mediated transfer of identity matching in pigeons (D'Amato, Salmon, \& Colombo, 1985 ) and why transfer between IM and CM tends to be bidirectional in pigeons.

Not that monkeys have a well-articulated concept of sameness. Monkeys that show evidence of the matching concept after IM training with colors or forms do not spontaneously apply the concept to flashing versus steady visual stimuli or to stimuli in other modalities. Considerable additional matching training with the new stimuli is necessary before such a demonstration is possible; it is as if the animals must develop the matching concept anew 
each time a significant change is made in the referent class of stimuli (see D'Amato, Salmon, \& Colombo, 1985). This limitation may be related to the fact that, in animals, irrelevant contextual cues gain stimulus control to an unusually strong degree. Possibly, pigeons are even more subject to such control than are monkeys.

The high accuracy on IM probe trials displayed by both the monkeys and the pigeons deserves comment. It indicates that in the CM/IM test cycle, even though the animals retrieved the correct comparison stimulus during the sample presentation period, they did not discard the information that they possessed concerning the identity of the sample. This raises an interesting question. Suppose probe trials were introduced after retention intervals of different durations. Would accuracy of the IM probe trials suffer more than accuracy on the CM baseline trials as the retention interval increased, which is what one might expect from a "directed forgetting" (of the sample stimulus) perspective? Or would the animals display a kind of parallel processing, retaining the sample stimulus to the same degree as its associated comparison stimulus? Finally, would important species differences emerge in the degree to which animals can maintain the identity of both stimuli?

More than 90 years ago, C. Lloyd Morgan (1894/1977) expressed serious reservations as to the ability of animals to perceive relations such as same/different. Although hindered by a lack of experimental data-which he himself lamented-his acute analysis is instructive even today. Still, the ape language-learning research of the last two decades makes it difficult not to ascribe a good measure of this capacity to the great apes. But it is also becoming evident that the ability to perceive abstract relations, or in somewhat more contemporary terminology, the capacity for rule-based or concept-based behavior, appears to suffer a sharp evolutionary gradient. Accurate description and analysis of this capacity in a variety of species is a major challenge for researchers in comparative cognition.

\section{REFERENCES}

Carter, D. E. \& Werner, T. J. (1978). Complex learning and information processing by pigeons: A critical analysis. Journal of the Experimental Analysis of Behavior, 29, 565-601.

D'Amato, M. R., \& Colombo, M. (1985). Auditory matching-to-sample in monkeys (Cebus apella). Animal Leaming \& Behavior, 13, 375-382.
D'Amato, M. R., \& SALmon, D. P. (1984). Cognitive processes in cebus monkeys. In H. L. Roitblat, T. G. Bever, \& H. S. Terrace (Eds.), Animal cognition (pp. 149-168). Hillsdale, NJ: Erlbaum.

D'Amato, M. R., Salmon, D. P., \& Colombo, M. (1985). Extent and limits of the matching concept in monkeys (Cebus apella). Journal of Experimental Psychology: Animal Behavior Processes, 11, 35-51.

D'Amato, M. R., Salmon, D. P., Loukas, E., \& Tomie, A. (1985). Symmetry and transitivity of conditional relations in monkeys $(\mathrm{Ce}$ bus apella) and pigeons (Columba livia). Journal of the Experimental Analysis of Behavior, 44, 35-47.

D'Amato, M. R., \& Worsham, R. W. (1974). Retrieval cues and shortterm memory in capuchin monkeys. Journal of Comparative and Physiological Psychology, 86, 274-282.

Herrenstein, R. J. (1985). Riddles of natural categorization. Philosophical Transactions of the Royal Society of London, B308, 129-144.

Holmes, P. W. (1979). Transfer of matching performance in pigeons. Journal of the Experimental Analysis of Behavior, 31, 103-114.

Honig, W. K., \& Thompson, R. K. R. (1982). Retrospective and prospective processing in animal working memory. In G. H. Bower (Ed.), The psychology of learning and motivation: Advances in theory and research (Vol. 16, pp. 239-283). New York: Academic Press.

KIRK, R. E. (1968). Experimental design: Procedures for the behavioral sciences. Belmont, CA: Brooks/Cole.

MILNER, A. D. (1973). Matching within and between sense modalities in the monkey (Macaca mulatta). Journal of Comparative \& Physiological Psychology, 83, 278-284.

MoRGAN, C. L. (1977). An introduction to comparative psychology. Washington, DC: University Publications of America. (Original work published 1894)

Premack, D. (1978). On the abstractness of human concepts: Why it would be difficult to talk to a pigeon. In S. H. Hulse, H. Fowler, \& W. K. Honig (Eds.), Cognitive processes in animal behavior (pp. 423-451). Hillsdale, NJ: Erlbaum.

Premack, D. (1983). The codes of man and beasts. Behavioral \& Brain Sciences, 6, 125-137.

SIEGEL, S. (1956). Nonparametric statistics for the behavioral sciences. New York: McGraw-Hill.

Wilson, B., Mackintosh, N. J., \& Boakes, R. A. (1985a). Matching and oddity learning in the pigeon: Transfer effects and the $a b-$ sence of relational learning. The Quarterly Journal of Experimental Psychology, 37B, 295-311.

Wilson, B., Mackintosh, N. J., \& Boakes, R. A. (1985b). Transfer of relational rules in matching and oddity learning by pigeons and corvids. The Quarterly Journal of Experimental Psychology, 37B, 313-332.

Zerbolo, D. J., JR. (1985). Categorical color coding in goldfish. Animal Learning \& Behavior, 13, 269-273.

Zerbolio, D. J., JR., \& Royalty, J. L. (1983). Matching and oddity conditional discrimination in the goldfish as avoidance responses: Evidence for conceptual avoidance learning. Animal Learning \& Behavior, 11, 341-348

(Manuscript received April 23, 1986; revision accepted for publication August 26, 1986.) 